Purdue University

Purdue e-Pubs

2013

\title{
Cyclic Operation of Molten-Salt Thermal Energy Storage in Thermoclines for Solar Power Plants
}

Z. Yang

Purdue University

S V. Garimella

Purdue University, sureshg@purdue.edu

Follow this and additional works at: http://docs.lib.purdue.edu/coolingpubs

Yang, Z. and Garimella, S V., "Cyclic Operation of Molten-Salt Thermal Energy Storage in Thermoclines for Solar Power Plants" (2013). CTRC Research Publications. Paper 185.

http://dx.doi.org/http://dx.doi.org/10.1016/j.apenergy.2012.09.043

This document has been made available through Purdue e-Pubs, a service of the Purdue University Libraries. Please contact epubs@purdue.edu for additional information. 


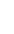

10

\title{
Cyclic Operation of Molten-Salt Thermal Energy Storage in Thermoclines for Solar Power Plants
}

\author{
Zhen Yang and Suresh V. Garimella* \\ Cooling Technologies Research Center, an NSF I/UCRC \\ School of Mechanical Engineering \\ 585 Purdue Mall, Purdue University \\ West Lafayette, Indiana 47907-2088 USA
}

* Submitted for publication in Applied Energy, Dec 2011, and in revised form, June 2012

** Author to whom correspondence should be addressed: (765) 494-5621, sureshg@purdue.edu 


\section{Abstract}

The cyclic operation of molten-salt thermal energy storage thermoclines for solar thermal power plants is systematically investigated. A comprehensive, two-temperature model is first developed for the cyclic operation of a thermocline operating with a commercially available molten salt as the heat transfer fluid and quartzite rock as the filler. Volume-averaged mass and momentum equations are employed, with the Brinkman-Forchheimer extension to the Darcy law used to model the porous-medium resistance. Energy equations for the molten salt and the filler are coupled by an interstitial Nusselt number representing the heat transfer between the phases. A finite-volume approach is employed to solve the governing equations. The model is validated against experiments from the literature and then used to systematically study the cyclic behavior of the thermocline thermal storage system. Thermal characteristics including temperature profiles and cycle efficiency are explored. Guidelines are developed for designing the dimensions and molten salt flow rates for solar thermocline systems of different power capacities. The cycle efficiency is found to be improved at smaller melt Reynolds numbers, larger length ratios (molten salt flow distance in a half-cycle to the filler particle diameter) and larger tank heights. The filler particle diameter and the tank volume are found to strongly influence the cycle efficiency.

Keywords: Solar thermal energy, energy storage, thermocline, molten salt, cyclic operation 


\section{Nomenclature}

$C_{P} \quad$ specific heat, $\mathrm{J} \cdot \mathrm{kg}^{-1} \mathrm{~K}^{-1}$

$d \quad$ diameter of thermocline tank, $\mathrm{m}$

$d^{\prime} \quad$ diameter of inlet and outlet ports, $\mathrm{m}$

$d_{s} \quad$ diameter of filler particles, $\mathrm{m}$

$\mathbf{e}_{\mathbf{r}} \quad$ unit vector in the $r$ direction, -

$\mathbf{e}_{\mathbf{x}} \quad$ unit vector in the $x$ direction, -

$\mathbf{e}_{\theta} \quad$ unit vector in the $\theta$ direction, -

F inertial coefficient, -

$g$

acceleration due to gravity, $\mathrm{m} / \mathrm{s}^{2}$

$h \quad$ thermocline tank height, $\mathrm{m}$

$h^{\prime} \quad$ distributor region height, $\mathrm{m}$

$h_{i} \quad$ interstitial heat transfer coefficient, $\mathrm{W} \cdot \mathrm{m}^{-3} \mathrm{~K}^{-1}$

K permeability, $\mathrm{m}^{2}$

$k \quad$ thermal conductivity, $\mathrm{W} \cdot \mathrm{m}^{-1} \mathrm{~K}^{-1}$

$p \quad$ pressure, $\mathrm{Pa}$

$T \quad$ temperature, $\mathrm{K}$

$t \quad$ time, $\mathrm{s}$

$t_{0} \quad$ half cycle period, $\mathrm{s}$

u velocity vector, $\mathrm{ms}^{-1}$

$u_{\text {mag }} \quad$ velocity magnitude, $\mathrm{ms}^{-1}$

$u_{0} \quad$ mean velocity magnitude at the inlet of the filler bed on top, $\mathrm{ms}^{-1}$ 


\section{Greek}

$\begin{array}{ll}\alpha & \text { thermal diffusivity of molten salt, } \mathrm{m}^{2} \mathrm{~s}^{-1} \\ \varepsilon & \text { porosity, - } \\ \mu & \text { viscosity of molten salt, } \mathrm{kgm}^{-1} \mathrm{~s}^{-1} \\ v & \text { kinematic viscosity of molten salt, } \mathrm{m}^{2} \mathrm{~s}^{-1} \\ \rho & \text { density, } \mathrm{kgm}^{-3} \\ \overline{\bar{\tau}} & \text { stress tensor, } \mathrm{Nm}^{-2} \\ \psi & \text { length ratio, } \psi=u_{0} t_{0} / d_{s}\end{array}$

\section{Subscripts}

$c \quad$ cold molten salt from the preheater

$h \quad$ hot molten salt from the collector field

$l \quad$ molten salt phase

$s \quad$ solid filler phase

$e \quad$ effective 


\section{$1 \quad 1$ Introduction}

The incorporation of thermal energy storage (TES) into the operation of parabolic-trough solar thermal power plants offers the potential to deliver electricity without fossil-fuel backup as well as to meet peak demand, independent of weather conditions. The current baseline design for Solar Energy Generating Systems (SEGS) uses synthetic oils such as Therminol VP-1 as the heat transfer fluid (HTF) in the collector field [1]. Therminol VP-1 has a low freezing point of $12^{\circ} \mathrm{C}$, and is stable up to $400^{\circ} \mathrm{C}$, which is higher in comparison to Caloria, the HTF used previously. However, use of Therminol VP-1 as the heat storage medium above $400^{\circ} \mathrm{C}$ in practical applications is difficult due to its high vapor pressure (>1 MPa). In order to improve the Rankine cycle efficiency to a level higher than $40 \%$, the desired storage medium must withstand temperatures above $450^{\circ} \mathrm{C}$ [2] while featuring low vapor pressures. Pressurization of the solar thermal power system for use with current synthetic oils above $400^{\circ} \mathrm{C}$ is cost-prohibitive.

Molten salts are likely ideal candidates as storage media with increased storage temperatures close to $500^{\circ} \mathrm{C}$ and extremely low vapor pressures. The higher storage temperatures achieved with molten salts can also reduce the volume of the storage tank for a given storage capacity. Moreover, molten salts are cheaper and more environmentally friendly than the currently used synthetic oils. The major disadvantage of molten salts is their relatively high melting temperature $\left(120^{\circ} \mathrm{C}\right.$ for HITEC XL [2], relative to $15^{\circ} \mathrm{C}$ for Caloria and $12^{\circ} \mathrm{C}$ for Therminol VP-1). This implies that special measures such as fossil-fuel or electric heating are necessary to 
maintain the salt at temperatures above the melting point to prevent solidification when solar power is unavailable or insufficient at night or in poor weather conditions. The fluidity of the molten salt in the loop may alternatively be maintained by discharging a small flow of hot molten salt from the TES unit through the loop during such periods.

A molten-salt TES thermocline uses only a single tank as the storage container and can thus potentially reduce costs significantly relative to a two-tank TES such as that used in the Solar Two demonstration plant [3,4]. In the thermocline, hot and cold regions of molten salt rely on buoyancy forces to maintain stable thermal stratification in a single tank. A low-cost filler material compatible with molten salts, such as quartzite rock [5], is used to occupy most of the volume of the thermocline and acts as the primary thermal storage material. This helps greatly reduce the amount of the relatively more expensive molten salt that is required in a two-tank storage system. It was shown in system studies [6,7] that a TES thermocline may offer the lowest-cost energy storage option, saving 35\% of cost compared to the two-tank system. A demonstration of such a thermocline at a pilot scale (2.3 MWh) was provided in Ref. [1].

A TES thermocline is a porous medium infiltrated with a heat transfer fluid (HTF). Thermal energy is transferred to the porous bed by the hot HTF flowing through the pores during a charge cycle and is released to a cold HTF during a discharge cycle. The heat exchange and energy storage in packed-bed porous media are important in many applications, and have attracted extensive research interest. 
1 Koh et al. [8,9] introduced the use of porous media for cooling high temperature

2 surfaces. Beasley and Clark [10] experimentally investigated the transient thermal

3 behavior of a packed bed composed of wires. Paek et al. [11] studied the cooling

4 characteristics of a porous medium in pulsating flows and found the cooling

5 effectiveness to increase with the amplitude of pulsation and the frequency. Singh et

6 al. [12] studied the transient thermal effects in a porous medium subject to oscillatory

7 flow of hot and cold fluid. The amplitude of temperature fluctuation in this study

8 varied significantly with distance and time, while the storage of energy was largely

9 governed by the fluid-to-solid phase thermal storage capacity ratio. El-Sebaii et al.

[13] investigated the year-round performance of a double-pass solar air heater with a

11 packed bed and reported an improvement in thermal performance, in terms of outlet temperature and thermohydraulic efficiency, relative to a system without a packed bed. Mawire et al. [14] developed a simplified one-dimensional single-phase model for an oil-pebble TES system to examine the thermal performance of three different pebble materials; a high ratio of the total exergy to the total energy stored was suggested as a good measure of thermal performance of the pebble material. A feedforward internal model control (IMC) structure for controlling the charging temperature of such a TES system for a solar cooker was also presented; thermal stratification was observed in the storage tank [15]. Fukai et al. [16] used carbon fibers to enhance the effective thermal conductivity of the storage medium in pebble-bed TES units. While heat transfer in packed-bed porous media has been widely studied, only limited investigations of the thermal performance of molten-salt thermocline TES 
1 have been reported despite its significance to solar thermal electricity generation.

2 Pacheco et al. [1] described the development of a molten-salt thermocline system and

3 compared it to a two-tank system; they suggested the molten-salt thermocline system

4 as a feasible option for thermal storage in a parabolic trough plant; the preliminary

5 thermal considerations in this work did not include parametric investigations of filler

6 size, tank diameter and tank height. Material properties were the focus of a few

7 studies $[2,5,17]$ of thermoclines, with no information on thermal performance

8 provided.

In a recent study, the discharge efficiency of thermal energy storage in thermocline tanks under an adiabatic wall condition was investigated for different melt flowrates, filler particle diameters and tank heights [18]. The influence of the interstitial heat transfer rate and the filler thermal conductivity were discussed in [19]. The effect of non-adiabatic boundaries on the thermal performance of thermocline tanks was explored in terms of the internal flow structure and resulting discharge efficiency in [20]. Thermal ratcheting of the tank wall caused by temperature variations and the settling of the filler particles was analyzed with a comprehensive thermomechanical model in [21]. Only the discharge of a thermocline system was considered in these works [18-21]; the temperature distribution and cycle efficiency under cyclic operation as a function of the operating conditions was not considered, and is the focus of the present study. This cyclic operation of a molten-salt thermocline for solar thermal electricity generation is distinct from previous studies of pulsating/oscillating flow conditions [10-12]; a molten-salt input/output flow at 
1 constant temperature levels is essential in the charge/discharge of a thermocline, as is

2 a narrow heat exchange zone [18] (also termed thermocline zone) in the storage tank.

3 There is a clear need for a theoretical model which predicts the cycle efficiency. So

4 also, physics-based guidelines for molten-salt thermocline design, which can be

5 scaled to different power capacities, are essential for applications in solar

6 thermal-electric power plants.

7 The present work aims to investigate the cyclic operation of a molten-salt TES

8 thermocline system for solar thermal-electric power plants, with a molten salt (HITEC)

9 used as the HTF and quartzite rock used as the filler. The thermal behavior, 10 including temperature profiles and cycle efficiency, are specifically investigated.

11 Based on the numerical results obtained, guidelines are provided for designing TES

12 thermoclines with various power capacities.

\section{Mathematical Modeling}

15

16

\subsection{Cyclic operation of a thermocline}

Figure 1 shows a schematic illustration of a parabolic-trough solar thermal power system equipped with a direct TES thermocline. The word "direct" means that the molten salt from the collector field flows through and exchanges heat energy directly with the filler material inside the thermocline without the need for a separate heat exchanger. The HTF considered here is the commercially available molten salt, HITEC, which is a eutectic mixture of the water-soluble, inorganic salts potassium nitrate $(53 \% \mathrm{wt})$, sodium nitrite $(40 \% \mathrm{wt})$ and sodium nitrate $(7 \% \mathrm{wt})$. It is in a 
1 liquid state above $149^{\circ} \mathrm{C}$ and very stable up to $538^{\circ} \mathrm{C}$. Its physical properties, such

2 as viscosity and thermal conductivity, change with temperature. HITEC is

3 nonflammable, non-explosive and releases no toxic vapors under recommended

4 conditions of use, and therefore is considered a good candidate as a molten-salt HTF

5 for use in parabolic-trough solar thermal electric plants.

The cyclic operation of a thermocline is considered here, with each cycle

7 consisting of a charge and a discharge process. During the charge half-cycle (left

8 side in Fig. 1), hot molten salt from the collector field flows via the top port into the

9 thermocline, decreases in temperature by transferring heat to the cold filler material, and finally exits the thermocline tank as a cooled liquid through the bottom port and

11 flows back to the collector field. In this manner, the thermal energy harnessed from

12 the collector field is stored in the filler material inside the thermocline tank. During

13 the discharge half-cycle (right side in Fig. 1), cold molten salt from the preheater unit

14 is pumped into the thermocline tank through the bottom port, heated by the filler 15 material that was heated in the previous charge half-cycle, and flows out of the upper port of the tank as hot molten salt. The thermal energy stored in the filler material is

17 thus retrieved by the molten salt HTF for use in steam generation.

The specific focus of the present work is on the cyclic operation of molten-salt

19 TES thermoclines. The structure of a TES thermocline is depicted in Fig. 2. The 20 thermocline tank of inner diameter $d$ is filled with a porous bed of quartzite rock of 21 height $h$. On both the top and bottom of the porous bed are distributor regions free 22 of filler material. These distributor regions serve to maintain a uniform flow 
1 condition at both ends of the filler bed in order to achieve good thermal stratification

2 inside the filler bed. Molten salt flows through the filler bed from the top to the

3 bottom of the filler bed in the charge half-cycle, and in the reverse direction during

4 the discharge half-cycle. The discharge and charge half-cycles need not be of the

5 same duration; however in the present work, they are assumed to be of equal duration

6 with no intervening dwell time. The half-cycle period $t_{0}$ is not limited to a value of

$7 \quad 12$ hours, and is determined by the application of interest.

\subsection{Governing equations}

For the axisymmetric coordinate system $(x-r)$ shown in Fig. 2, dimensional

11 governing equations for continuity, momentum and energy are presented in Ref. [18];

12 the corresponding non-dimensional governing equations for the laminar flow and heat 13 transfer in a porous filler bed are as follows:

14 a. Continuity equation:

$$
\varepsilon \frac{\partial\left(\Phi_{\rho}\right)}{\partial \tau}+\nabla \cdot\left(\Phi_{\rho} \mathbf{U}\right)=0
$$

15

b. Momentum equation:

$$
\begin{aligned}
& \operatorname{Re} \Psi \frac{\partial\left(\Phi_{\rho} \mathbf{U}\right)}{\partial \tau}+\operatorname{Re} \Psi \nabla \cdot\left(\frac{\Phi_{\rho} \mathbf{U}}{\varepsilon}\right)=-\varepsilon \nabla P+\nabla \cdot \mathbf{T} \\
& +\varepsilon \Phi_{\rho} G r \mathbf{e}_{\mathbf{x}}-\varepsilon\left(\frac{\Phi_{\mu} \mathbf{U}}{D a^{2}}+\frac{F \operatorname{Re} \Psi}{D a} \Phi_{\rho}|\mathbf{U}| \mathbf{U}\right)
\end{aligned}
$$

c. Energy equation for the molten salt: 


$$
\begin{aligned}
& \operatorname{Pr} \operatorname{Re} \frac{\partial}{\partial \tau}\left(\varepsilon \Phi_{\rho} \Phi_{C p l} \Theta_{l}\right)+\operatorname{Pr} \operatorname{Re} \nabla \cdot\left(\Phi_{\rho} \Phi_{C p l} \Theta_{l} \mathbf{U}\right) \\
& =\frac{1}{\Psi} \nabla \cdot\left(\Phi_{k e} \nabla \Theta_{l}\right)+2 \operatorname{Pr} A \operatorname{Re} \Phi_{\mu}\left[\mathbf{S} \cdot \mathbf{S}^{\prime}+\operatorname{tr}(\mathbf{S}) \operatorname{tr}\left(\mathbf{S}^{\prime}\right)\right]+\Phi_{k l} N u_{i} \Psi\left(\Theta_{s}-\Theta_{l}\right)
\end{aligned}
$$

1 d. Energy equation for the filler material:

$$
\operatorname{Pr} \operatorname{Re} \frac{\partial}{\partial \tau}\left[(1-\varepsilon) \Omega \Phi_{\rho s} \Phi_{C p s} \Theta_{s}\right]=-\Phi_{k l} N u_{i} \Psi\left(\Theta_{s}-\Theta_{l}\right)
$$

2 where $\mathbf{T}=2 \mathbf{S}-\frac{2}{3} \operatorname{tr}(\mathbf{S}), \quad \mathbf{S}=\frac{\nabla \mathbf{U}+(\nabla \mathbf{U})^{T}}{2}$ and $\mathbf{S}^{\prime}=\frac{\nabla(\mathbf{U} / \varepsilon)+[\nabla(\mathbf{U} / \varepsilon)]^{T}}{2}$, and the

3 other terms are defined elsewhere under Nomenclature.

The non-dimensional parameters included in Eqs. (1) - (4) are defined as follows

$5 \tau=\frac{t}{t_{0}}, \quad \mathbf{U}=\frac{\mathbf{u}}{u_{0}}, \nabla=\mathbf{e}_{\mathbf{x}} \frac{\partial}{\partial X}+\mathbf{e}_{\mathbf{r}} \frac{\partial}{\partial R}+\frac{\mathbf{e}_{\boldsymbol{\theta}}}{R} \frac{\partial}{\partial \theta}, X=\frac{x}{u_{0} t_{0}}, R=\frac{r}{u_{0} t_{0}}, \quad R e=\frac{u_{0} d_{s}}{v_{h}}$,

$6 \quad P=\frac{p t_{0}}{\mu_{h}}, \Theta=\frac{T-T_{c}}{T_{h}-T_{c}}, \quad G r=\frac{g u_{0} t_{0}^{2}}{v_{h}}, \quad D a=\frac{\sqrt{K}}{u_{0} t_{0}}, \quad \operatorname{Pr}=\frac{v_{h}}{\alpha_{h}}, \quad A=\frac{u_{0}^{2}}{C p_{l, h}\left(T_{h}-T_{c}\right)}$,

$7 \quad N u_{i}=\frac{h_{i} d_{s}^{2}}{k_{l, h}}, \psi=\frac{u_{0} t_{0}}{d_{s}}$. The interstitial Nusselt number $N u_{i}$ thermally couples the

8 molten salt with the filler medium. The parameter $\Psi$ represents the ratio of the

9 distance covered by the molten salt flow in a charge (or discharge) half-cycle to the diameter of the filler particles; this parameter is hereafter referred to as length ratio.

11 Coefficients $\Phi_{\rho}, \Phi_{\mu}, \Phi_{C p l}$, and $\Phi_{k l}$, account for the temperature dependence of the 12 density, viscosity, specific heat, and thermal conductivity of the molten salt, respectively; $\Phi_{k e}, \Phi_{\rho s}$ and $\Phi_{C p s}$ are the corresponding coefficients for the effective thermal conductivity of the molten salt-filler combination, and the density and specific heat of the filler material, respectively. These coefficients are well represented by the following expressions, with a deviation of less than $1 \%$ from the experimental data in Ref. [22]: 
1

$$
\Phi_{\rho}=1-\frac{0.732\left(T_{h}-T_{c}\right)}{2084.4-0.732 T_{c}} \Theta_{l}
$$

$$
\Phi_{\mu}=\frac{\exp \left\{-4.343-2.0143 \ln \left[\left(T_{h}-T_{c}\right) \Theta_{l}+T_{c}\right]+10.094\right\}}{\exp \left(-4.343-2.0143 \ln T_{c}+10.094\right)}
$$

$$
\Phi_{k l}=\frac{-6.53 \times 10^{-4}\left[\left(T_{h}-T_{c}\right) \Theta_{l}+T_{c}\right]+0.5908}{-6.53 \times 10^{-4} T_{c}+0.5908}
$$

$$
\Phi_{C p l}=1, \quad \Phi_{C p s}=1, \Phi_{\rho s}=1
$$

Parameter $\Phi_{k e}$ is obtained by

$$
\Phi_{k e}=\Phi_{k l} \frac{1+2 \beta \phi+\left(2 \beta^{3}-0.1 \beta\right) \phi^{2}+\phi^{3} 0.05 \exp (4.5 \beta)}{1-\beta \phi}
$$

where $\phi=1-\varepsilon$ and $\beta=\left(k_{s}-k_{l}\right) /\left(k_{s}+2 k_{l}\right)$ [23]. The filler bed is treated as being composed of spherical rock particles of uniform diameter. According to Wakao et al. [24], the interstitial Nusselt number for a liquid particle bed can be expressed as

$$
N u_{i}=6(1-\varepsilon)\left(2+1.1 \operatorname{Re}_{L}^{0.6} \operatorname{Pr}_{L}^{1 / 3}\right)
$$

where $\operatorname{Re}_{L}$ and $\operatorname{Pr}_{L}$ are the local Reynolds and Prandtl numbers, respectively.

Assuming that the distributor regions are properly designed so that uniform flows are achieved at the inlet and outlet of the filler bed [18], and the molten salt temperatures from the collector field $T_{h}$ and from the preheater $T_{c}$ are constant, the boundary conditions may be specified as follows:

a) At the top exit of the filler bed, molten salt at the temperature of the upstream flow exits the tank in the discharge period; in the charge period, on the other hand, molten salt at $T_{h}\left(\Theta_{l}=1\right)$ from the collector field flows into the tank. Therefore, the nondimensional flow and thermal conditions are given as: 
In the discharge half-cycle $(0<\tau<1): \quad \vec{U}=\vec{e}_{x}, \quad \partial \Theta_{l} / \partial X=0$

In the charge half-cycle $(1<\tau<2): \vec{U}=-\vec{e}_{x}, \Theta_{l}=1$

1 b) At the bottom exit of the filler bed, molten salt at $T_{c}\left(\Theta_{l}=0\right)$ from the preheater

2 enters the tank during the discharge period, while molten salt at the upstream flow

3 temperature exits the tank in the charge period. In this case, the nondimensional

4 flow and thermal conditions are given as:

In the discharge half-cycle $(0<\tau<1): \partial \vec{U} / \partial X=0, \Theta_{l}=0$

In the charge half-cycle $(1<\tau<2): \partial \vec{U} / \partial X=0, \partial \Theta_{l} / \partial X=0$

5 In Eqs. (6) and (7), flow boundary conditions at the top exit of the filler bed are set

6 equal in value, i.e., $|\vec{U}|=1$, but are reversed in direction during the discharge and

7 charge half cycles, since the volumes of hot molten salt flowing into and out of the

8 tank are identical. The flow velocities at the bottom exit are not fixed, but rather are

9 determined from a volume balance in the tank during the calculation; the derivative of

10 velocity in the flow direction is set to zero, i.e., $\partial \vec{U} / \partial X=0$ in Eqs. (8) and (9),

11 representing fully developed flow. Equations (1) - (5) show that heat transfer and

12 fluid flow in a thermocline storage tank are governed by $R e, \Psi$ and material properties,

13 i.e., $\Phi_{\rho}, \Phi_{\mu}, \Phi_{k l}, \Phi_{k s}, \Phi_{k e}, \Phi_{C p l}, \Phi_{C p s}$ and $\operatorname{Pr}$. Other parameters, such as $G r, D a$ and $F$,

14 may affect the pressure distribution in the momentum equation, but have no influence

15 on fluid flow and heat transfer inside the filler bed as long as the distributors maintain

16 a uniform flow in the transverse direction and thermal stratification is achieved.

17 Once the molten salt and the filler material are selected, i.e., the material properties

18 are determined, the characteristics of thermocline thermal energy storage are solely 
1 determined by $R e$ and $\Psi$, provided that good flow distributors are in place.

\section{$2 \quad 2.3$ Solution Procedure}

The computational domain is discretized into finite volumes. Values of all the variables at the center of the square mesh cells are stored. A second-order upwind scheme is used for the convective fluxes, while a central-differencing scheme is used for discretizing the diffusion fluxes, as in [18]. A second-order implicit scheme is used for time discretization. Pressure-velocity coupling is implemented through the PISO algorithm [25]. Iterations at each time step are terminated when the dimensionless residuals for all equations drop below $10^{-4}$. The computations are performed using the commercial software package, FLUENT 6.1 [26]. User-defined functions (UDFs) are developed to account for Eqs. (3) and (4). Grid and time-step independence is verified by evaluating the results with various grid densities and time intervals. Settings of $\Delta X=\Delta R=0.01$ and $\Delta \tau=1.25 \times 10^{-5}$ are chosen for the work presented here, as this case resulted in a temperature along the line $r=0$ throughout the whole discharge process which differs by less than $1 \%$ relative to results from a finer mesh and time step of $\Delta X=\Delta R=0.005$ and $\Delta \tau=6.16 \times 10^{-6}$.

The numerical method was previously validated against experimental results from a pilot-scale TES thermocline [1]; satisfactory agreement of axial temperature profiles at different discharge times was achieved between the model and the experimental results [18].

\section{Results and Discussion}


The thermal and hydrodynamic conditions at the beginning of an operating cycle

2 in the thermocline are not known a priori. The initial conditions are thus arbitrarily

3 chosen to be a uniform high-temperature $(\Theta=1)$ and zero velocity $(\mathbf{U}=0)$

4 everywhere in the domain. The calculation is carried out for many cycles until a

5 periodic state is achieved: the temperature and velocity profiles along the axis $(r=0)$

6 at the start of a discharge-charge cycle deviate less than $0.01 \%$ from the previous

7 cycle under these conditions. A true cyclic operation is then deemed to have been

8 reached. The typical number of cycles required for achieving this cyclic operation is

9 approximately 50.

Figure 3(a) shows the temperatures in both the molten salt and the filler during

11 one discharge-charge cycle in a thermocline of non-dimensional height $H=1.0 C_{0}\left(C_{0}\right.$

12 is non-dimensional parameter defined in Eq. (11), and will be discussed later).

13 During the discharge half-cycle $(0<\tau<1)$, thermal energy stored in the filler material

14 is retrieved by the cold molten-salt flow, and thus the temperature is higher in the

15 filler than in the molten salt. In the charge process $(1<\tau<2)$, on the other hand, hot molten salt is pumped through the upper port (at $X=1.0 C_{0}$ ) into the thermocline to heat the cold filler, and thus the temperature of the molten salt is higher than that of

18 the filler, as shown in Fig. 3(a). The temperature difference between the molten salt 19 and the filler decreases sharply with a decrease in the Reynolds number and an 20 increase in the length ratio $\Psi$ or the tank height $H$; under these conditions, the 21 temperature profiles in the molten salt and the filler are nearly indistinguishable in the 22 plots. For clarity of illustration, the filler temperature profiles are omitted from the 
1 rest of the figures (which feature smaller $R e$, larger $\Psi$ and $H$ ) in this work.

\section{$2 \quad 3.1$ Temperature profiles}

Although the thermocline zone may be disturbed in some cases [27], it is very stable under the current calculation conditions. With ideal flow distributors at the top and bottom of the filler bed, the thermal and hydrodynamic behavior of thermoclines was shown in [18] to be quite uniform in the transverse direction (at any horizontal plane along the height of the tank) throughout the porous filler bed; thus, the temperature profile along the axis $(r=0)$ provides a complete representation of the temperature distribution in the thermocline. Typical temperature profiles along the axis $(r=0)$ approximately halfway through the discharge and charge half-cycles are shown in Fig. 4(a) at $\tau=0.5$ (discharge) and $\tau=1.5$ (charge). Three distinct zones are identified in the temperature profiles: a constant high-temperature $(\Theta=1)$ zone, a constant low-temperature $(\Theta=0)$ zone, and a heat-exchange zone with a changing temperature $(0<\Theta<1)$. In the constant high-temperature $(\Theta=1)$ and low-temperature $(\Theta=0)$ zones, the molten salt and the filler are in thermal equilibrium with no net heat transfer between these phases; in the heat-exchange zone $(0<\Theta<1)$, heat is transferred from the filler to the molten salt in the discharge half-cycle (and in the opposite direction during the charge half-cycle) [18]. The heat-exchange zone travels from the bottom to the top of the filler bed in the discharge half-cycle and reverses course in the charge half-cycle. The propagation velocity of the heat-exchange zone can be predicted, as shown in [18], by 


$$
v=\frac{C_{P, l, h} \rho_{l, h} u_{0}}{\varepsilon C_{P, l, h} \rho_{l, h}+(1-\varepsilon) C_{P, s, h} \rho_{s, h}}
$$

1 This velocity is a function only of material properties and filler-bed porosity. During

2 the time of a charge or discharge half-cycle $t_{0}$, the distance over which the

3 heat-exchange zone can travel is $v t_{0}$; this distance provides a measure of the tank

4 height required for a charge or discharge process. If a thermocline tank is shorter

5 than $v t_{0}$, it will have run out of thermal energy before the discharge half-cycle ends.

6 Alternatively, it will have already been fully charged part way through the charge

7 half-cycle, leading to significant under-utilization of the available energy from the

8 collector field. Thermocline tanks with heights larger than $v t_{0}$ are therefore

9 recommended for improved efficiency in sequestering thermal energy from the

10 collector field. The effects of tank height will be further considered later in this

11 discussion.

The axial temperature distributions shown in Fig. 4 illustrate a discharge-charge

13 cycle for a length ratio $\Psi$ of 450 at different $R e$. The non-dimensional tank height $H$

14 is set at $1.5 C_{0}$ for the plots in this figure, where $C_{0}$ is the non-dimensional form of the

15 front-propagation distance $v t_{0}$, and is defined as

$$
C_{0}=\frac{v t_{0}}{u_{0} t_{0}}=\frac{C_{P, l, h} \rho_{l, h}}{\varepsilon C_{P, l, h} \rho_{l, h}+(1-\varepsilon) C_{P, s, h} \rho_{s, h}}
$$

16 Just as in the case of the propagation velocity, $C_{0}$ also depends only on material 17 properties and the filler-bed porosity. As shown in the figure, the heat-exchange zone is longer at the larger Reynolds number; for instance, it extends more widely from $0.35 C_{0}$ to $1.3 C_{0}$ at $R e=240$ (Fig. 4 (a) $\tau=0.5$ ), relative to that from $0.4 C_{0}$ to 
2 high-temperature zone decreases in extent with an increase in the Reynolds number;

3 at $\tau=0.05$, for example, this zone extends from $0.8 C_{0}$ to $1.5 C_{0}$ at $R e=240$ compared

4 to its extent from $0.65 C_{0}$ to $1.5 C_{0}$ at $R e=10$.

Figure 3 shows the temperature profiles for two different tank heights. It is clear that the shorter tank $\left(H=1.0 C_{0}\right.$ in Fig. 3(a) $)$ has sharper temperature gradients in

7 the molten salt and a correspondingly shorter heat-exchange zone due to the proximity

8 of the hot and cold ports, compared to a longer tank $\left(H=1.5 C_{0}\right.$ in Fig. 3(b)). As

9 stated earlier, the inlet molten-salt temperatures for the charge $\left(T_{h}\right)$ and discharge $\left(T_{c}\right)$ half-cycles are fixed in these calculations. A shorter heat-exchange zone provides a

11 smaller distance for the molten salt to be completely heated or cooled, which results 12 in a larger temperature difference between the filler and the melt and therefore, 13 greater heat transfer rates between the phases. For instance, under conditions of $R e$ $14=240$ and $\Psi=150$, the temperature difference between the molten salt and the filler 15 is about 0.035 at $H=1.5 C_{0}$, and takes a larger value of 0.05 at $H=1.0 C_{0}$. The larger temperature difference results in a larger generation of entropy $\Delta S$, defined (for the 17 discharge half-cycle) as

$$
\Delta S=\frac{T_{s}-T_{l}}{T_{s} T_{l}} Q
$$

where $T_{s}$ and $T_{l}$ is the temperatures of the filler and molten salt $\left(T_{s}>T_{l}\right)$, respectively, and $Q$ is the amount of heat exchanged between the filler and molten salt. A larger entropy generation is responsible for a more significant loss in the quality (i.e., temperature) of the available thermal energy. A similar conclusion can be drawn for 
1 the charge half-cycle. This implies that the amount of high-quality thermal energy

2 that can be retrieved at a high temperature level during a cyclic operation would be

3 reduced. Cycle efficiency will be discussed further later in this paper. As an

4 illustration, the molten-salt temperature at the upper port $\left(X=1.0 C_{0}\right)$ of the short $(H=$

$\left.51.0 C_{0}\right)$ tank in Fig. 3(a) at the end of the discharge process $(\tau=1)$ is only 0.35 ,

6 relative to the value of 0.7 at the upper port $\left(X=1.5 C_{0}\right)$ of the tall $\left(H=1.5 C_{0}\right)$ tank in

7 Fig. 3(b); this demonstrates the faster drop in temperature of the output molten-salt

8 flow to a lower quality in the shorter tank.

The Reynolds number has a similar effect on performance as the tank height: increasing the Reynolds number increases the temperature difference between the 11 molten salt and the filler, increase entropy generation, and decrease the thermal quality of the output energy. For instance, the molten-salt temperature at the upper port $\left(X=1.5 C_{0}\right)$ at the end of discharge $(\tau=1)$ is 0.8 at $\operatorname{Re}=240$ (Fig. 4(a)), and 0.9 at $\operatorname{Re}=10$ (Fig. 4(b)). conditions of $\operatorname{Re}=240$ and $H=1.5 C_{0}$, the heat-exchange zone for $\Psi=450$ extends from $0.35 C_{0}$ to $1.3 C_{0}$ (see Fig. 4(a) $\tau=0.5$ ), while at $\Psi=150$ it spans a wider extent 19 from $0.2 C_{0}$ to $1.5 C_{0}$ (see Fig. 3(b) $\tau=0.5$ ). Since $\Psi$ is the ratio of the flow distance of molten salt in a discharge/charge half-cycle to the diameter of the filler particles, a

21 large $\Psi$ means that the molten salt can traverse a larger number of filler particles during the time of discharge/charge, and hence exchange heat energy with more filler 
1 particles before it flows out of the tank; the heat-exchange zone decreases in extent

2 with a corresponding increase in the constant high-temperature zone. For example,

3 for $R e=240$ and $H=1.5 C_{0}$, the constant high temperature zone at $\Theta=1$ extends from

$4 \quad 0.8$ to 1.5 at $\tau=0.05$ for $\Psi=450$ (Fig. 4(a)), compared to the range from 1.0 to 1.5 at

$5 \tau=0.05$ for $\Psi=150$ (Fig. 3(b)). The temperature of output molten-salt flow at the

6 end of the discharge half-cycle $(\tau=1)$ increases with $\Psi$, as shown by a comparison at

$7 \quad X=1.5 C_{0}$ and $\tau=1$ of the temperatures at $\Psi=450(\Theta=0.8$ in Fig. 4(a)) and that at $\Psi$

$8=150(\Theta=0.7$ in Fig. 3(b)).

Abrupt changes in the temperature profiles near the ports may be experienced during transitions between the charge and discharge processes, as shown in Fig. 4

11 from $\tau=1$ to 1.05 (from discharge to charge) and from $\tau=2$ to 0.05 (from charge to 12 discharge). These abrupt changes are attributed to the cyclic operation of 13 thermoclines in a solar thermal power system. At the end of the discharge half-cycle $14(\tau=1)$, the molten-salt temperature at the upper port may have already dropped below $15 \Theta=1$ (see Fig. 4 at $\tau=1$ and $X=1.5 C_{0}$ ); at the same time, fresh hot molten salt at $\Theta$ $16=1$ from the collector field begins to be pumped through the upper port. The sudden direct contact between the two molten salt streams at different temperatures causes a large temperature gradient near the upper port $\left(X=1.5 C_{0}\right)$ at the beginning of a charge 19 half-cycle, as shown in Fig. 4 at $\tau=1.05$. For a similar reason, a larger temperature 20 gradient near the lower port $(X=0)$ also occurs at the beginning of a discharge 21 half-cycle $(\tau=0.05)$. These abrupt changes in temperature can accelerate the deterioration in the thermal quality of the heat energy for two reasons: a greater 
1 gradient of temperature in the molten salt induces a greater generation of entropy, as

2 does the greater temperature difference between the fresh, hot (or cold) molten salt

3 and the filler at the beginning of the discharge (or charge) half-cycle. The transition

4 becomes more abrupt as measured by the extent of temperature change at the upper

5 port at $\tau=1$, from discharge to charge, or that at the lower port at $\tau=2$ (also the start

6 of the next cycle, $\tau=0$ ), from charge to discharge, as $R e$ increases and $\Psi$ decreases.

7 For instance, the change in temperature at the transition from discharge to charge ( $\tau=$

8 1) is 0.2 at $R e=240$, and 0.1 at $R e=10$, as shown in Fig. 4; similarly, it is 0.27 at $\Psi=$

$9 \quad 150$ (Fig. 3(b)), and 0.2 at $\Psi=450$ (Fig. 4(a)).

The tank height also affects the temperature transition significantly. For

11 instance, the change in temperature between half-cycles is 0.65 at $H=1.0 C_{0}$, while it is reduced to 0.27 at $H=1.5 C_{0}$, as shown in Fig. 3.

\subsection{Output temperature history}

The thermal energy delivered from the collector field is of high quality, i.e., a high temperature level $(\Theta=1)$. After it is stored in, and then retrieved from, the thermocline, the quality of thermal energy may deteriorate, and the output molten-salt temperature could experience a corresponding drop. Figure 5 shows the output temperature history under different conditions for two tank heights. As shown in the figure, the output temperature drops faster with an increase in the Reynolds number and a decrease in the length ratio $\Psi$, as shown in both Figs. 5(a) and (b). As previously discussed, increasing the Reynolds number or decreasing the length ratio $22 \Psi$ can significantly extend the heat-exchange zone, reduce the constant high 
1 temperature zone, and shorten the period of high temperature output of the molten-salt

2 flow. For instance, in a thermocline tank of height $1.0 C_{0}$, the output temperature at

$3 R e=240$ and $\Psi=150$ drops below 0.95 at $\tau=0.6$, much earlier than at $R e=10$ and

$4 \Psi=450$ for which this drop occurs at $\tau=0.87$. The tank height $H$ also has a

5 significant influence on the output temperature. A taller tank $\left(H=1.5 C_{0}\right)$ has a more

6 prolonged period of high-temperature output of molten salt than does a shorter tank

$7 \quad\left(H=1.0 C_{0}\right): \quad$ for $R e=240$ and $\Psi=150$, the time period with output molten salt at a

8 temperature higher than 0.95 is $\tau=0.6$ for $H=1.0 C_{0}$, and $\tau=0.7$ for $H=1.5 C_{0}$, as

9 shown in Fig. 5.

The reasons for this behavior of the output temperature lie in the temperature

11 profiles analyzed in section 3.1 above: increasing the Reynolds number, decreasing the length ratio $\Psi$ and shortening the tank height $H$ can increase the temperature difference between molten salt and filler as well as incur a more abrupt change in temperature at the transition between the charge and discharge half-cycles, thus 15 increasing the entropy generation and ultimately reducing the quality of the output heat energy.

In practical applications, it is desired that most of the stored thermal energy be retrieved at a high temperature level in order to meet the design conditions for generating superheated steam in the turbines; the higher quality also helps maintain higher thermal-to-electrical conversion efficiency in the turbine generator. As a result, only thermal energy with temperature above a certain level is considered "useful" energy. In this work, molten salt flows with $\Theta_{l}>0.95$ are considered as 
1 providing useful energy as shown in Fig. 5. Heat energy retrieved below $\Theta_{l}=0.95$

2 as in the later stages of the discharge half-cycle contribute to a waste of heat energy.

\subsection{Cycle efficiency}

It is of interest to quantify the amount of useful energy that a thermocline system can deliver during its cyclic operation. The cycle efficiency of a thermocline thermal energy storage system is defined in this work as follows

$$
\eta=\frac{\text { Heat energy retrieved with } \Theta_{l}>\Theta_{0} \text { in a discharge half cycle }}{\text { Heat energy delivered from the collector field in a charge half cycle }}
$$

where $\Theta_{0}$ is a threshold value determined by the application of interest. As stated above, a value of 0.95 for $\Theta_{0}$ is chosen in this work, implying that thermal energy delivered at temperatures greater than $\left(T_{c}+0.95\left(T_{h}-T_{c}\right)\right)$ qualifies as useful energy. If $T_{h}=450^{\circ} \mathrm{C}$ and $T_{c}=250^{\circ} \mathrm{C}$, for example, HITEC liquid delivered at temperatures above $440^{\circ} \mathrm{C}$ is considered useful for generating superheated steam in the steam turbine.

The cycle efficiency is intimately related to the output temperature history, which is a function of $R e, \Psi$ and $H$, as discussed earlier. Three representative values for $H$, i.e., $H=0.5 C_{0}, 1.0 C_{0}$ and $1.5 C_{0}$, are considered in this discussion for illustration of the influence of $\operatorname{Re}$ and $\Psi$. Figure 6 presents the cycle efficiencies under different conditions. As shown in the figure, the cycle efficiency monotonically decreases with an increase in Reynolds number and increases with the length ratio $\Psi$ for all tank heights. At a given $R e$ and $\Psi$, the cycle efficiency is highest for the tallest tank at $H$ $=1.5 C_{0} . \quad$ For example, at $R e=240$ and $\Psi=450, \eta$ is 0.86 at $H=1.5 C_{0}$ and drops to $\eta=0.38$ at $H=0.5 C_{0}$. 
2 the following correlation:

$$
\eta=a(\ln R e)^{2}+b \ln \operatorname{Re}+c
$$

3 where the coefficients $a, b$ and $c$ are written as

$$
\begin{gathered}
\text { At } H=1.5 C_{0}: a=5.4 \times 10^{-6} \Psi-7.02 \times 10^{-3} \\
b=8.83 \times 10^{-10} \Psi^{3}-1.04 \times 10^{-6} \Psi^{2}+4.11 \times 10^{-4} \Psi-5.12 \times 10^{-2} \\
c=-9.5 \times 10^{-8} \Psi^{2}+1.59 \times 10^{-4} \Psi+0.909 \\
\text { At } H=1.0 C_{0}: a=-1.42 \times 10^{-8} \Psi^{2}+1.4 \times 10^{-5} \Psi-7.56 \times 10^{-3} \\
b=7.42 \times 10^{-10} \Psi^{3}-7.36 \times 10^{-7} \Psi^{2}+2.61 \times 10^{-4} \Psi-3.86 \times 10^{-2} \\
\quad c=-5 \times 10^{-7} \Psi^{2}+4.66 \times 10^{-4} \Psi+0.782 \\
\text { At } H=0.5 C_{0}: a=1.45 \times 10^{-9} \Psi^{2}+2 \times 10^{-7} \Psi-2.22 \times 10^{-3} \\
b=6.33 \times 10^{-10} \Psi^{3}-6.72 \times 10^{-7} \Psi^{2}+2.5 \times 10^{-4} \Psi-3.74 \times 10^{-2} \\
c=-1.18 \times 10^{-7} \Psi^{2}+1.33 \times 10^{-4} \Psi+0.412
\end{gathered}
$$

4 The good agreement between the correlation and the numerical results is shown in Fig.

5 6. Equation (14) is valid over the ranges $10<R e<250$ and $150<\Psi<450$.

It is observed from the figure that the cycle efficiency of thermoclines with $H=$ $0.5 C_{0}$ is significantly lower than that at the other two heights $\left(H=1.5 C_{0}\right.$ and $\left.1.0 C_{0}\right)$. In short thermocline tanks with $H<1.0 C_{0}$, the heat-exchange zone arrives at the lower (upper) port in a discharge (charge) half-cycle prior to the end of the half-cycle, so that the tank is already fully charged (depleted) with thermal energy partway through a half-cycle. This causes short thermoclines to no longer accept (output) thermal energy in the latter part of the half-cycles, which results in poor cycle efficiencies. 


\subsection{Design procedure and examples}

The non-dimensional results obtained in the previous sections can be conveniently used to study the cyclic operation of different thermocline TES units. In this section, the utility of Eq. (14) to predict cycle efficiencies for different thermocline designs is demonstrated. The following procedure for predicting the efficiency of a thermocline with a charge/discharge power $P$ and a cycle period $2 t_{0}$ is proposed:

1. Choose tank diameter $d$ and filler particle diameter $d_{s}$ based on practical constraints.

2. Calculate the cross-sectional area of the storage tank $A=0.25 \pi d^{2}$, and then the superficial velocity $u_{0}$ of molten salt, $u_{0}=P /\left(A \rho_{l, h} C_{P, l, h}\left(T_{h}-T_{c}\right)\right)$.

3. Calculate $R e$ and $\Psi$ using $R e=u_{0} d_{s} / v$ and $\Psi=u_{0} t_{0} / d_{s}$.

4. Calculate the cycle efficiency $\eta$ using Eq. (14), according to the selected non-dimensional tank height $H\left(H=0.5 C_{0}, 1.0 C_{0}\right.$ or $\left.1.5 C_{0}\right)$.

5. Calculate the dimensional height of the tank $h$ as $h=d_{S} H$.

Table I lists some design examples for thermoclines with equal diameter and height, i.e., $d=h$. As shown in the table, the methodology proposed above can be applied to the study of thermoclines with various scales. The power range considered here is from 100 to $1000 \mathrm{MW}$, as shown in the table. Thermoclines designed with $H=1.5 C_{0}$ show larger cycle efficiencies than do those with $H=1.0 C_{0}$. For example, a thermocline of $500 \mathrm{MW}$ capacity and a cycle period of 24 hours has a cycle efficiency of $84.0 \%$ at $H=1.5 C_{0}$, and $76.1 \%$ at $H=1.0 C_{0}$, although the former 
1 has a dimensions $(45.0 \mathrm{~m}) 15 \%$ larger than that of the latter $(39.3 \mathrm{~m}) . \quad$ A thermocline

2 with a larger power has higher the cycle efficiency. For example, a thermocline with

3 a power of $1000 \mathrm{MW}$ has a cycle efficiency $\left(85.3 \%\right.$ at $H=1.5 C_{0}$ and $77.3 \%$ at $H=$

$\left.41.0 C_{0}\right)$ greater than that of $100 \mathrm{MW}$ power $\left(80.3 \%\right.$ at $H=1.5 C_{0}$ and $72.4 \%$ at $H=$ $5 \quad 1.0 C_{0}$ ) by $5 \%$.

\subsection{Parameter influences}

In this section, the influence of filler particle diameter and tank diameter on the cycle efficiency of thermoclines is specifically explored, since it is the first of the five-step procedure proposed above for thermocline design.

A thermocline of $500 \mathrm{MW}$ capacity and an operation cycle period of $24 \mathrm{~h}$ is considered for this discussion. Figure 7 shows cycle efficiencies of thermoclines with different shapes. As the thermocline becomes flatter, with an increased $d / h$ ratio, its cycle efficiency decreases. Tall thermoclines with small $d / h$ can achieve higher cycle efficiencies. However, practical considerations may limit thermocline tanks to $d / h \approx 1$ in terms of tank integrity as well as reduced heat loss. From the figure, it is clear that at $d / h=1$, a thermocline with $H=1.0 C_{0}$ has a tank diameter of $39.3 \mathrm{~m}$ and a corresponding cycle efficiency of $76 \%$, while that with $H=1.5 C_{0}$ has a tank diameter of $45 \mathrm{~m}$ and a higher cycle efficiency of $84 \%$. For a specific power and cycle duration, while the cycle efficiency increases with tank volume, tank volume cannot be arbitrarily increased. Instead, a tradeoff between increased cycle efficiency and construction and operating costs is necessary; such a study is beyond the scope of the present discussion but is being conducted in ongoing work. 
2 shown in Fig. 8. Reducing the filler particle diameter can increase the total interface

3 area between the filler material and molten salt, and thus raise the interstitial heat

4 transfer rate. A higher interstitial heat transfer rate can effectively decrease the

5 temperature difference between filler and molten salt at a given overall heat transfer

6 rate, and thus decrease the corresponding entropy generation. As a result, the quality

7 of output thermal energy increases with a decrease in the filler particle diameter as

8 does the cycle efficiency. As seen from Fig. 8, the cycle efficiency increases from

$960 \%$ to $74 \%$ as the filler particle diameter decreases from 0.2 to $0.1 \mathrm{~m}$ for a thermocline of tank diameter $45 \mathrm{~m}$ and $H=1.0 C_{0}$ (the dimensional tank height $h$ is 30

$11 \mathrm{~m})$. Also evident from the figure is that thermoclines with smaller tank diameters,

12 for example $d=35 \mathrm{~m}$, have higher cycle efficiencies than those with larger tank 13 diameters. Taller thermoclines also show larger cycle efficiencies as previously 14 discussed.

\section{Conclusions}

A two-temperature model is developed for investigating cyclic operation of thermocline thermal energy storage systems using molten salt as the heat transfer fluid and inexpensive quartzite rock as the filler. The influence of important parameters, including the Reynolds number, length ratio (ratio of molten-salt flow distance in a half-cycle to the filler particle diameter) and non-dimensional tank height, are specifically explored. The non-dimensional results are applicable for the study of 
1 thermoclines of various thermal capacities and under different operating conditions.

Cycle efficiency of thermocline storage tanks is well predicted by the correlation developed in Eq. (14) for $10<R e<250$ and $150<\Psi<450$. The cycle efficiency is found to increase with a decrease in $R e$ and an increase in $\Psi$. Taller tanks also result in greater cycle efficiencies.

Procedures are developed for the design of thermocline storage tanks of various power capacities. The use of smaller filler particles can greatly increase the cycle efficiency. For example, a thermocline of $P=500 \mathrm{MW}, t_{0}=12 \mathrm{~h}, d=45 \mathrm{~m}$ and $h=$ $30 \mathrm{~m}$ has a cycle efficiency of $74.3 \%$ for a filler particle diameter of $0.1 \mathrm{~m}$, compared to $60.4 \%$ with larger particles of diameter $0.2 \mathrm{~m}$. In a thermocline at specific power and cycle period, increasing the tank volume can significantly improve the cycle efficiency. For example, at $d / h=1$, a thermocline of $P=500 \mathrm{MW}$ and $t_{0}=12 \mathrm{~h}$ has a cycle efficiency of $76 \%$ with tank volume of $4.77 \times 10^{4} \mathrm{~m}^{3}(d=h=39.3 \mathrm{~m})$, while it has a higher efficiency of $84 \%$ with tank volume of $7.16 \times 10^{4} \mathrm{~m}^{3}(d=h=45 \mathrm{~m})$.

\section{References}

1 J.E. Pacheco, S.K. Showalter, W.J. Kolb, Development of a molten-salt thermocline thermal storage system for parabolic trough plants, J. Sol. Energ-T ASME 124 (2002) 153-159.

2 D. Kearney, U. Herrmann, P. Nava, Assessment of a molten salt heat transfer fluid in a parabolic trough solar field, J. Sol. Energ-T ASME 125 (2003) 170-176.

3 J.E. Pacheco, R. Gilbert, Overview of recent results of the solar two test and 
evaluations program, Paper number RAES99-7731. In: Hogan R, Kim Y, Kleis S, O’Neal D, Tanaka T, editors. Renewable and advanced energy systems for the $21 \mathrm{st}$ century. Proceedings of the 1999 ASME International Solar Energy Conference, Maui, HI, April 11-14. 1999 [CD-Rom].

4 G.J. Kolb, V. Hassani, Performance analysis of thermocline energy storage proposed for the $1 \mathrm{MW}$ SAGUARO solar trough plant, Paper number ISEC2006-99005. Proceedings of ISEC 2006 ASME International Solar Energy Conference, Denver, CO, July 8-13, 2006.

5 D. Brosseau, J.W. Kelton, D. Ray, M. Edgar, Testing of thermocline filler materials and molten-salt heat transfer fluids for thermal energy storage systems in parabolic trough power plants, J. Sol. Energ-T ASME 127 (2005) 109-116.

6 H. Price, A parabolic trough solar power plant simulation model, http://www.nrel.gov/docs/fy03osti/33209.pdf, last accessed 19 July 2010.

7 D. Kearney, Engineering evaluation of a molten salt HTF in a parabolic trough solar field, NREL Contract No. NAA-1-30441-04, 2001.

8 J.C.Y. Koh, R. Colony, Analysis of cooling effectiveness for porous material in a coolant passage. ASME J. Heat Transfer 96 (1974) 324-330.

9 J.C.Y. Koh, R.L. Stevens, Enhancement of cooling effectiveness by porous materials in coolant passage. ASME J. Heat Transfer 97 (1975) 309-311.

10 D.E. Beasley, J.A. Clark, Transient response of a packed bed for thermal energy storage. Int. J. Heat Mass Transfer 27 (1984) 1659-1669.

11 J.W. Paek, B.H. Kang, J.M. Hyun, Transient cool-down of a porous medium in 
pulsating flow, Int. J. Heat Mass Transfer 42 (1999) 3523-3527.

12 C. Singh, R. G. Tathgir, K. Muralidhar, Energy storage in fluid saturated porous media subjected to oscillatory flow, Heat Mass Transfer (2009) 45:427-441.

13 A.A. El-Sebaii, S. Aboul-Enein, M.R.I. Ramadan, E. El-Bialy, Year round performance of double pass solar air heater with packed bed, Energy Conversion and Management 48 (2007) 990-1003.

14 A. Mawire, M. McPherson, R.R.J. van den Heetkamp, S.J.P. Mlatho, Simulated performance of storage materials for pebble bed thermal energy storage (TES) systems, Appl. Energy 86 (2009) 1246-1252.

15 A. Mawire, M. McPherson, A feedforward IMC structure for controlling the charging temperature of a TES system of a solar cooker, Energy Conversion and Management 49 (2008) 3143-3154.

16 J. Fukai, M. Kanou, Y. Kodama, O. Miyatake, Thermal conductivity enhancement of energy storage media using carbon fibers, Energy Conversion and Management $41(2000)$ 1543-1556.

17 S. Guillot, A. Faik, A. Rakhmatullin, J. Lambert, E. Veron, P. Echegut, C. Bessada, N. Calvet, X. Py, Corrosion effects between molten salts and thermal storage material for concentrated solar power plants, Appl. Energy 94 (2012) 174-181.

18 Z. Yang, S.V. Garimella, Thermal analysis of solar thermal energy storage in a molten-salt thermocline, Sol. Energy 84 (2010) 974-985.

19 C. Xu, Z. Wang, Y. He, X. Li, F. Bai, Sensitivity analysis of the numerical study on the thermal performance of a packed-bed molten salt thermocline thermal 
storage system, Appl. Energy 92 (2012) 65-75.

20 Z. Yang, S.V. Garimella, Molten-salt thermal energy storage in thermocline under different environmental boundary conditions, Appl. Energy 87 (2010) 3322-3329.

21 S. Flueckiger, Z. Yang, S. V. Garimella, An integrated thermal and mechanical investigation of molten-salt thermocline energy storage, Appl. Energy 88 (2011) $2098-2105$.

22 HITEC heat transfer salt, Costal Chemical Co., L.L.C., Brenntag Company, available at http://www.coastalchem.com/, last accessed 19 July 2010.

23 E.E. Gonzo, Estimating correlations for the effective thermal conductivity of granular materials, Chem. Eng. J. 90 (2002) 299-302.

24 N. Wakao, S. Kaguei, Heat and mass transfer in packed beds, Gordon and Breach Science Publishers, New York, 1982.

25 R.I. Issa, Solution of implicitly discretized fluid flow equations by operator splitting, J. Comput. Phys. 62 (1986) 40-65.

26 Fluent 6.3 User's Guide, ANSYS Inc., Lebanon, NH (2006).

27 F.G.F. Qin, X.P. Yang, Z. Ding, Y. Zuo, Y. Shao, R. Jiang, X.X. Yang, Thermocline stability criterions in single-tanks of molten salt thermal energy storage, Appl. Energy (2012) in press. 


\section{Figure captions}

Figure 1 A parabolic-trough solar thermal power system equipped with a direct TES thermocline. A molten salt is the HTF in the loop system.

Figure 2 Schematic illustration of a TES thermocline in axisymmetric coordinates (image of the rocks from http://www.concretematerialscompany.com/products/5eighthx.jpg).

Figure 3 Molten-salt temperature along the axis $(r=0)$ in thermoclines of different tank heights during a discharge-charge cycle $(R e=240, \Psi=150)$ : (a) $H=$ $1.0 \mathrm{C} 0$, and (b) $H=1.5 \mathrm{C} 0$. A more abrupt change in temperature along the axis is observed for the shorter thermocline.

Figure 4 Molten salt temperature along the axis $(r=0)$ during a discharge-charge cycle $(H=1.5 \mathrm{C} 0)$ : (a) $R e=240, \Psi=450$, and (b) $R e=10, \Psi=450 . \quad$ The heat-exchange zone $(0<\Theta<1)$ expands and the constant-temperature zone $(\Theta=0$ or 1$)$ shrinks at a larger Reynolds number.

Figure 5 Output temperature history of molten salt during the discharge half-cycle: (a) $H=1.0 \mathrm{C} 0$, and (b) $H=1.5 \mathrm{C} 0$. The temperature drops faster with an increase in the Reynolds number $R e$ and a decrease in the length ratio $\Psi$. A longer tank $(H=1.5 \mathrm{C} 0)$ can effectively delay the drop of the output temperature, compared to a shorter tank $(H=1.0 \mathrm{C} 0)$.

Figure 6 Cycle efficiencies under different conditions for different tank heights: (a) $H$ $=1.5 \mathrm{C} 0,(\mathrm{~b}) H=1.0 \mathrm{C} 0$, and (c) $H=0.5 \mathrm{C} 0 . \quad \eta$ decreases with an increase in $R e$ and a decrease in $\Psi . \quad \eta$ in a taller thermocline $(H=1.5 \mathrm{C} 0)$ is significantly larger than that in a shorter one $(H=0.5 \mathrm{C} 0)$. The correlation shows good agreement with the numerical data. 
Figure 7 Influence of tank diameter on the cycle efficiency (500 MW power, $24 \mathrm{~h}$ cycle, $0.1 \mathrm{~m}$ filler particle diameter). As tank diameter $d$ increases (the thermocline becomes flatter), the cycle efficiency monotonically drops. Thermoclines with $H=1.5 \mathrm{C} 0$ shows a higher efficiency than those with $H=$ $1.0 \mathrm{C} 0$.

Figure 8 Influence of filler particle diameter on cycle efficiency in thermoclines of $500 \mathrm{MW}$ power and $24 \mathrm{~h}$ cycle. The cycle efficiency decreases with an increase in filler particle diameter. Taller thermoclines with a smaller tank diameter $(d=35 \mathrm{~m})$ have higher cycle efficiencies. 


\section{Tables}

Table I. Results for various representative thermocline designs with $d=h, t_{0}=12 \mathrm{~h}$ and filler particle diameter $d_{s}$ of $0.1 \mathrm{~m}$.

\begin{tabular}{cccccccc}
\hline & \multicolumn{2}{c}{$100 \mathrm{MW}$} & \multicolumn{2}{c}{$500 \mathrm{MW}$} & \multicolumn{2}{c}{$1000 \mathrm{MW}$} \\
\cline { 2 - 7 }$H$ & $d$ or $h(\mathrm{~m})$ & $\eta(-)$ & $d$ or $h(\mathrm{~m})$ & $\eta(-)$ & $d$ or $h(\mathrm{~m})$ & $\eta(-)$ \\
\hline $1.0 C_{0}$ & 23.0 & $72.4 \%$ & 39.3 & $76.1 \%$ & 49.6 & $77.3 \%$ \\
$1.5 C_{0}$ & 26.3 & $80.3 \%$ & 45.0 & $84.0 \%$ & 56.7 & $85.3 \%$ \\
\hline
\end{tabular}




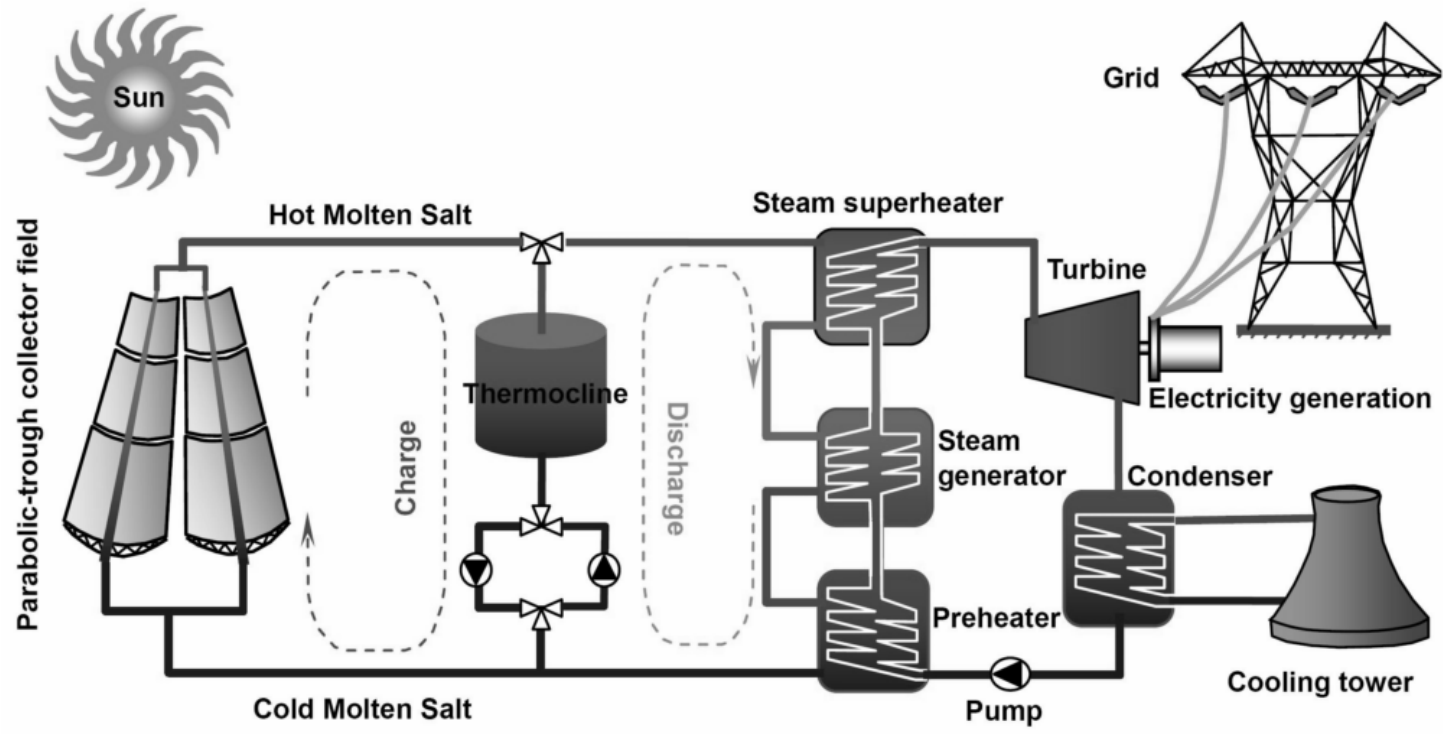

Figure 1 A parabolic-trough solar thermal power system equipped with a direct TES thermocline. A molten salt is the HTF in the loop system. 


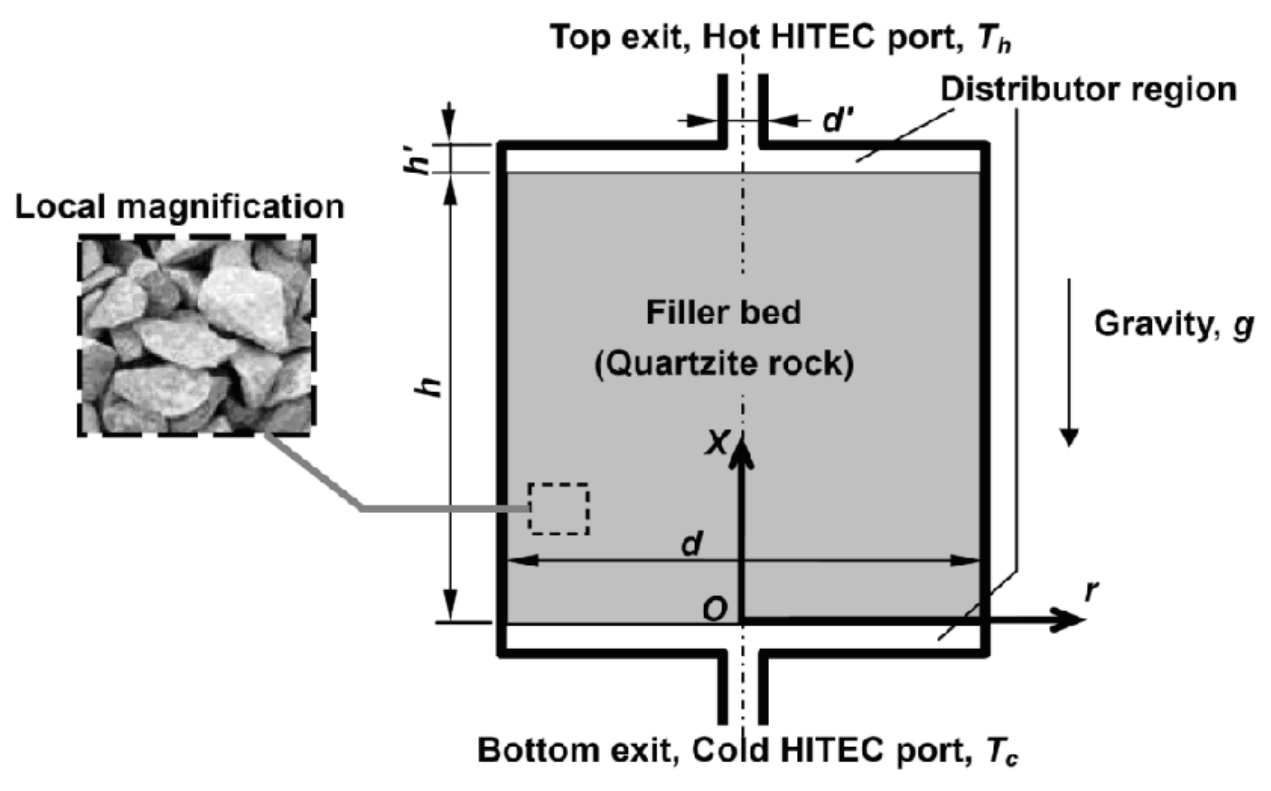

Figure 2 Schematic illustration of a TES thermocline in axisymmetric coordinates (image of the rocks from http://www.concretematerialscompany.com/products/5eighthx.jpg). 


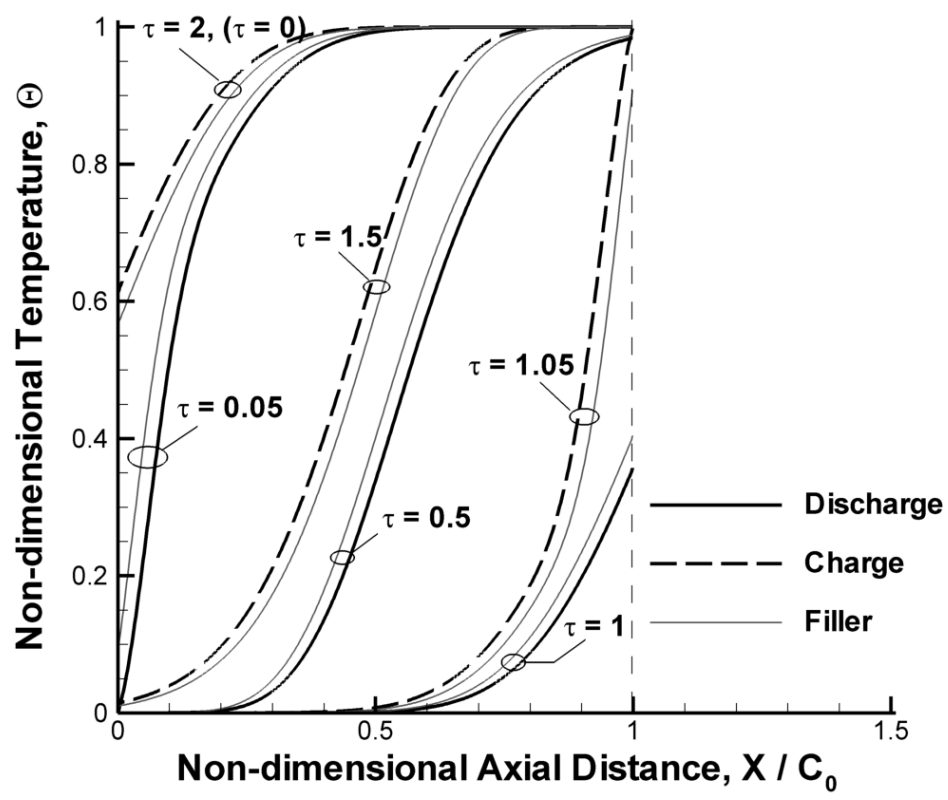

(a)

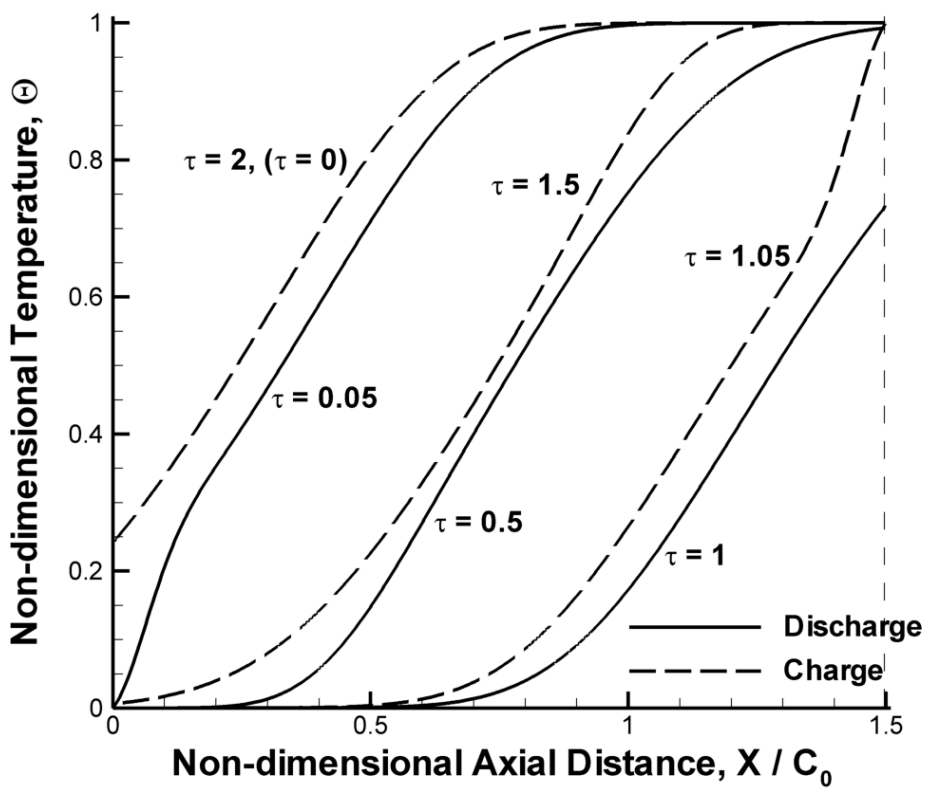

(b)

Figure 3 Molten-salt temperature along the axis $(r=0)$ in thermoclines of different tank heights during a discharge-charge cycle $(R e=240, \Psi=150)$ : (a) $H=1.0 C_{0}$, and (b) $H=1.5 C_{0} . \quad$ A more abrupt change in temperature along the axis is observed for the shorter thermocline. 


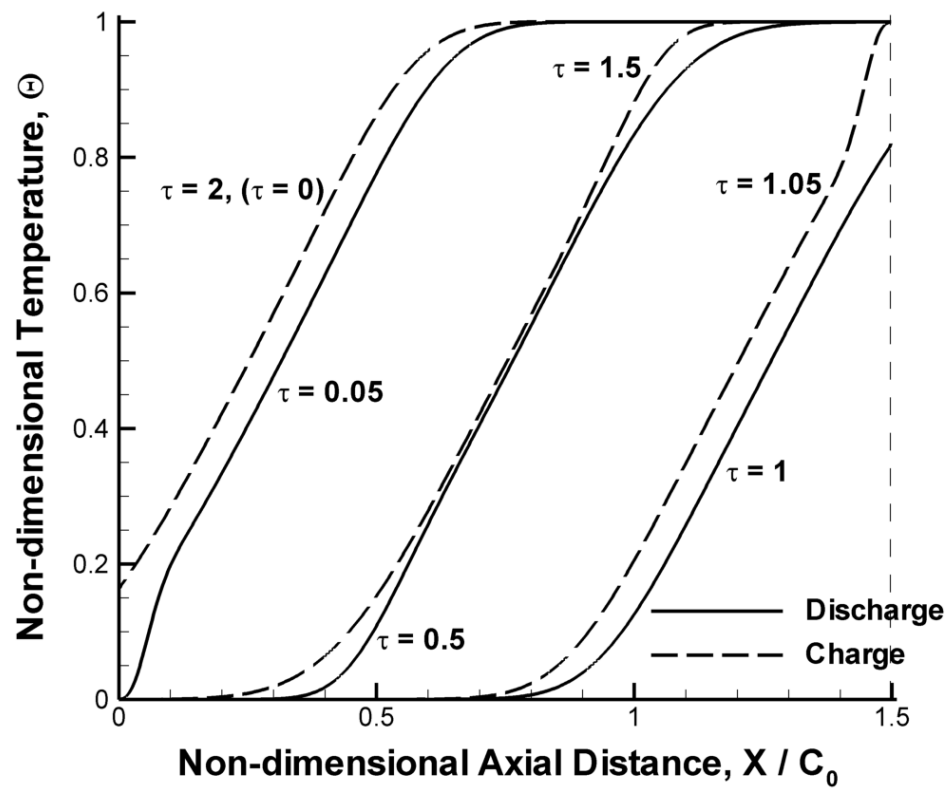

(a)

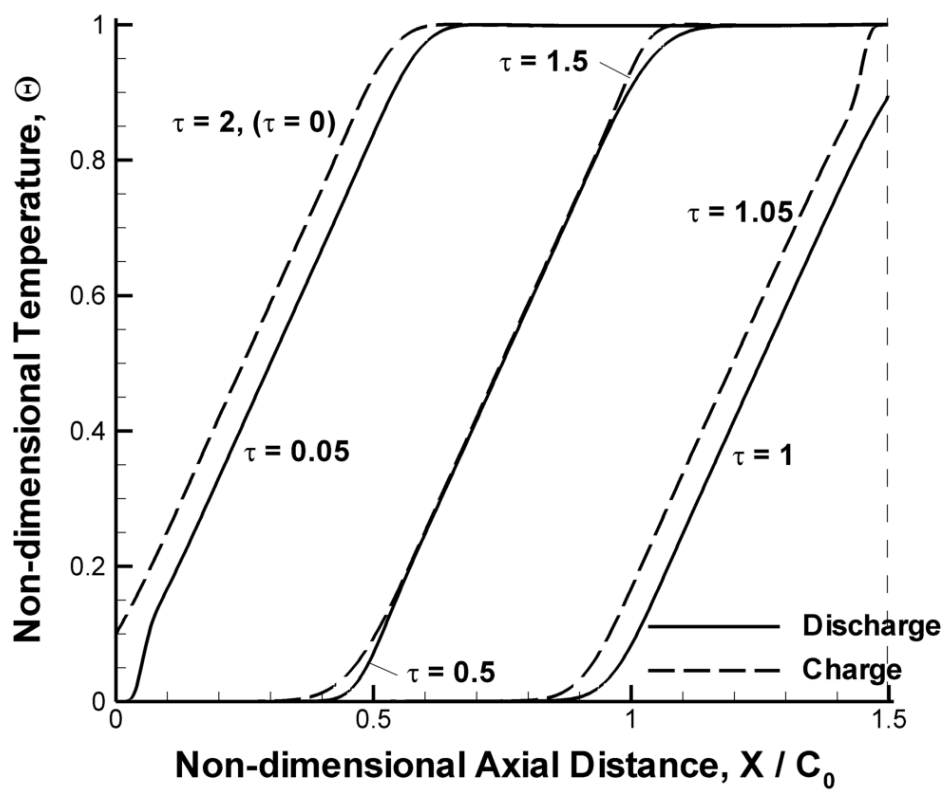

(b)

Figure 4 Molten salt temperature along the axis $(r=0)$ during a discharge-charge cycle $\left(H=1.5 C_{0}\right)$ : (a) $R e=240, \Psi=450$, and (b) $R e=10, \Psi=450$. The heat-exchange zone $(0<\Theta<1)$ expands and the constant-temperature zone $(\Theta=0$ or 1) shrinks at a larger Reynolds number. 


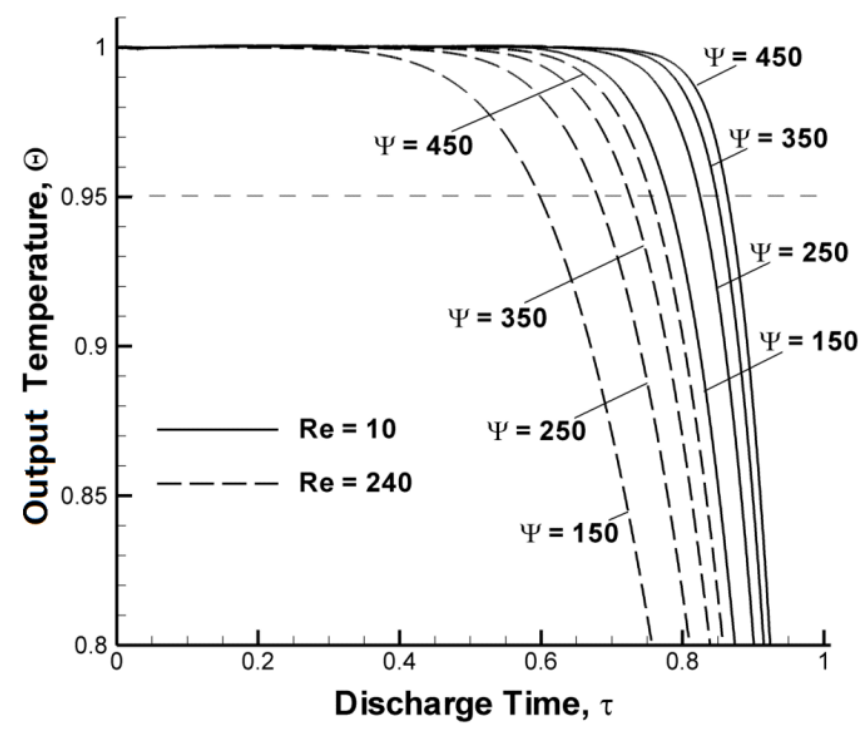

(a)

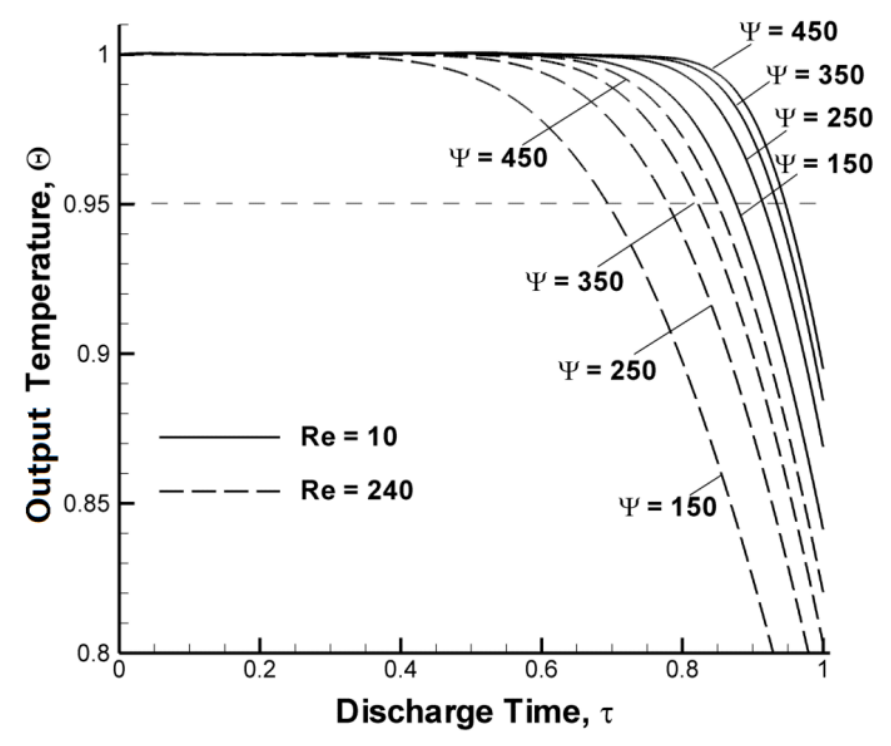

(b)

Figure 5 Output temperature history of molten salt during the discharge half-cycle: (a) $H=1.0 C_{0}$, and (b) $H=1.5 C_{0}$. The temperature drops faster with an increase in the Reynolds number $R e$ and a decrease in the length ratio $\Psi$. A longer tank $\left(H=1.5 C_{0}\right)$ can effectively delay the drop of the output temperature, compared to a shorter tank

$$
\left(H=1.0 C_{0}\right)
$$




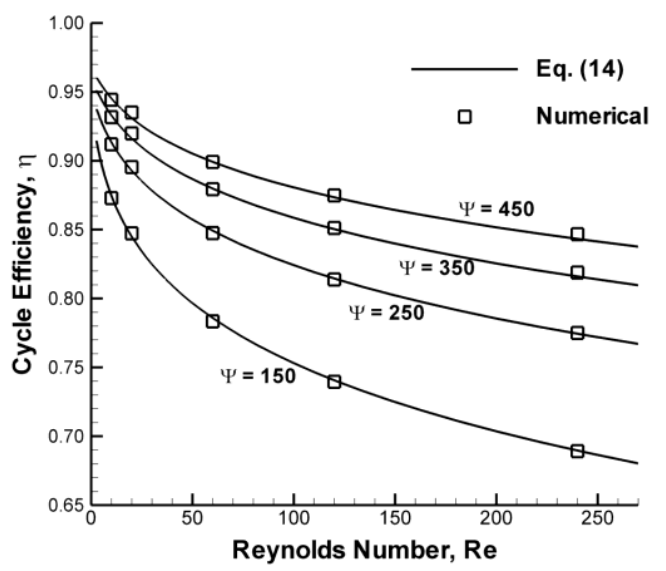

(a)

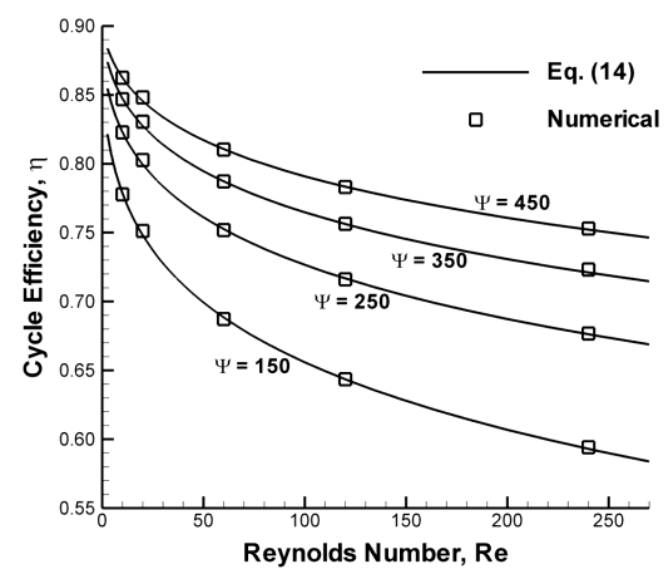

(b)

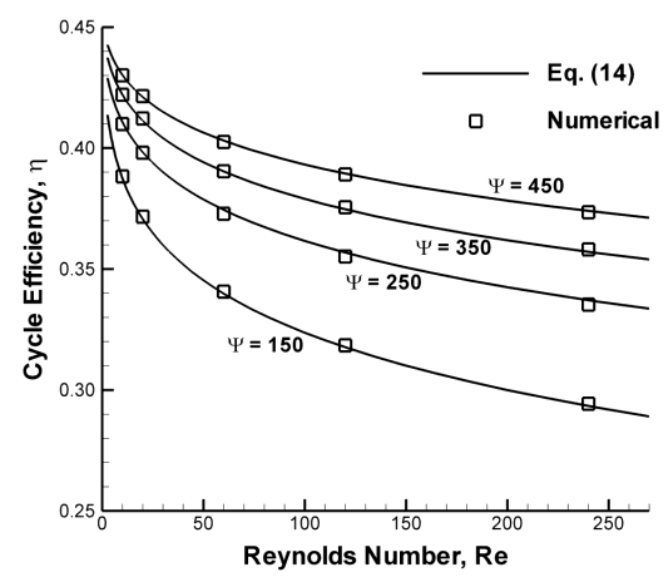

(c)

Figure 6 Cycle efficiencies under different conditions for different tank heights: (a) $H=1.5 C_{0}$, (b) $H=1.0 C_{0}$, and (c) $H=0.5 C_{0} . \quad \eta$ decreases with an increase in $R e$ and a decrease in $\Psi . \quad \eta$ in a taller thermocline $\left(H=1.5 C_{0}\right)$ is significantly larger than that in a shorter one $\left(H=0.5 C_{0}\right)$. The correlation shows good agreement with the numerical data. 


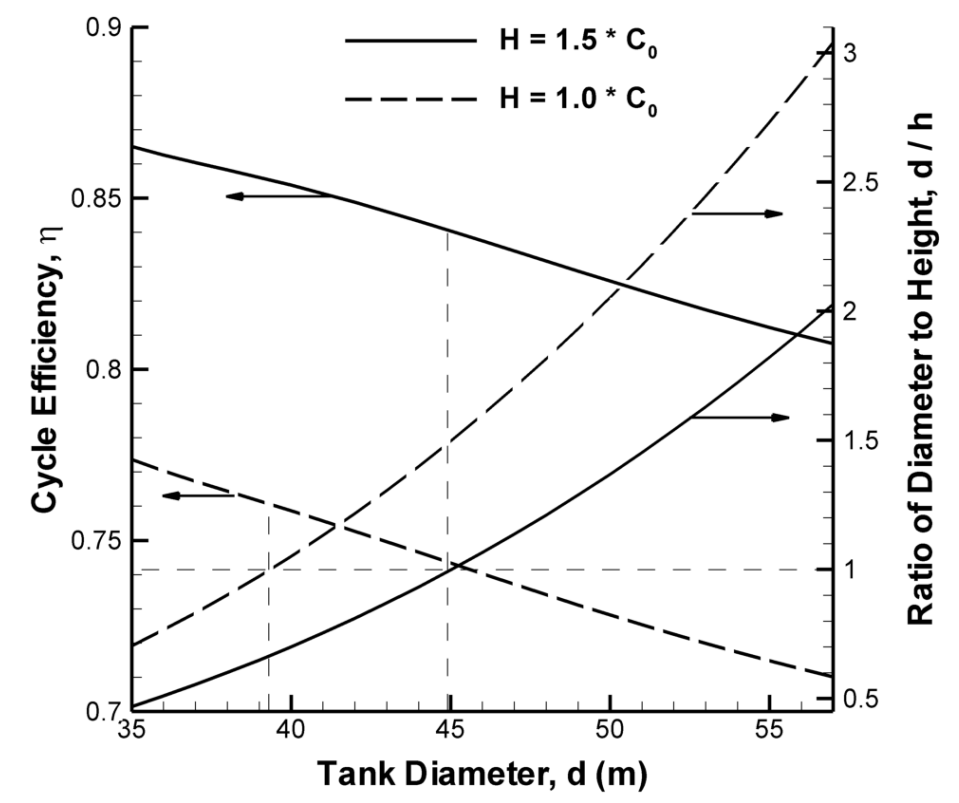

Figure 7 Influence of tank diameter on the cycle efficiency (500 MW power, $24 \mathrm{~h}$ cycle, $0.1 \mathrm{~m}$ filler particle diameter). As tank diameter $d$ increases (the thermocline becomes flatter), the cycle efficiency monotonically drops. Thermoclines with $H=$ $1.5 C_{0}$ shows a higher efficiency than those with $H=1.0 C_{0}$. 


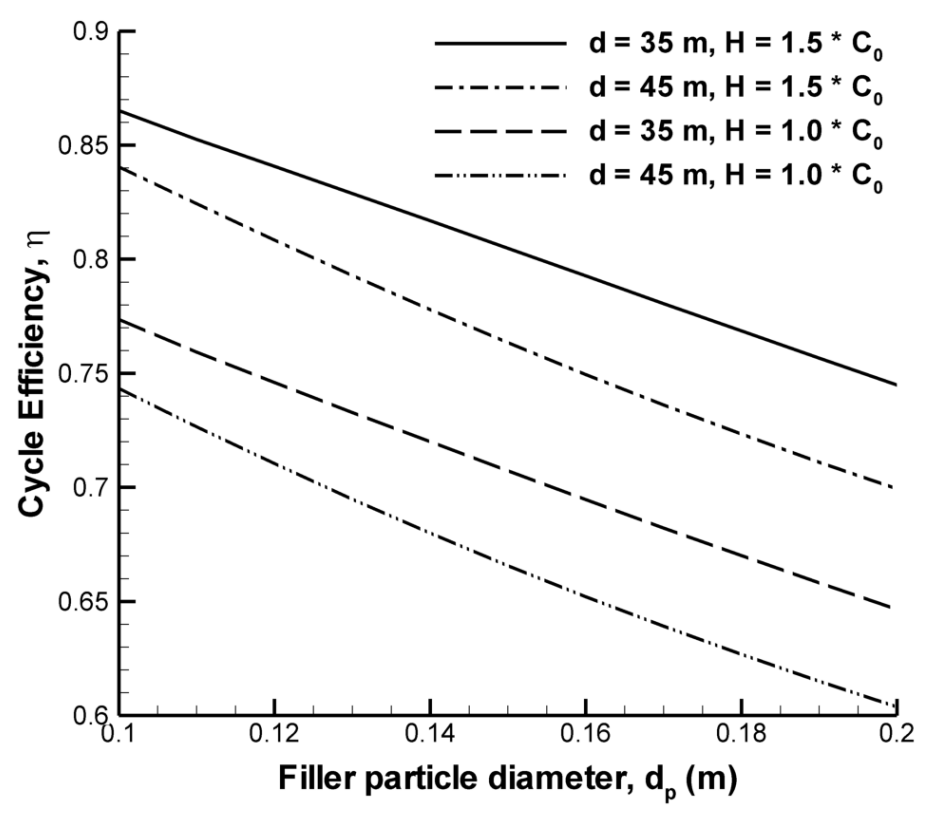

Figure 8 Influence of filler particle diameter on cycle efficiency in thermoclines of $500 \mathrm{MW}$ power and $24 \mathrm{~h}$ cycle. The cycle efficiency decreases with an increase in filler particle diameter. Taller thermoclines with a smaller tank diameter $(d=35 \mathrm{~m})$ have higher cycle efficiencies. 